\title{
Outer Shock Interaction in Young Core-Collapse SNRs
}

\author{
Jae-Joon Lee \\ Korea Astronomy and Space Science Institute, Daejeon 305-348, Korea \\ email: leejjoon@kasi.re.kr
}

\begin{abstract}
Studying the environments in which core-collapse supernovae (SNe) explode and then subsequently evolve is essential to establish the nature of the mass loss and the explosion of the progenitor star. The spatial structure of the outer shock in young core-collapse SNRs provides an opportunity to study the nature of the medium into which the remnant has been expanding. We present our X-ray study of the outer shocks in young core-collapse SNRs in our Galaxy. For Cas A and G292.0+1.8, we find that both remnants have been likely interacting with dense red supergiant winds. For other remnants with bright thermal X-ray emission from the shell, we suggest that they are interacting with pre-existing circumstellar structure. We discuss the nature of the winds and the progenitor stars.
\end{abstract}

Keywords. ISM: individual (Cas A) — supernova remnants - X-rays: ISM — stars: winds

\section{Young Core-Collapse SNRs expanding inside their RSG Winds.}

Stellar mass is the most important parameter that determines the structure, the evolution and the fate of stars. Observations have established that stars with initial mass of $8-15 \mathrm{M}_{\odot}$ undergo core collapse during their red supergiant (RSG) phases and produce Type II-P SNe (Smartt et al. 2009). On the other hand, the fate of more massive stars is still not well established (see the discussion in Smith et al. 2011). For example, stars in $15-30 \mathrm{M}_{\odot}$ may explode during the RSG phase, producing Type II-L, IIb, or IIn SNe while stars $>30 \mathrm{M}_{\odot}$ may evolve into Wolf-Rayet stars and produce Type Ib or Ic SNe. But observational constraints are rather indirect and weak. Also, their fate likely become complicated by their binary interaction.

For a star that undergoes SN explosion during the RSG phase (or soon after it evolved past the RSG phase), its remnant will primarily interact with the surrounding RSG wind. Existence of such an RSG wind has been suggested for SN 1987A (Chevalier \& Emmering 1989; Sugerman et al. 2005). The extent of the RSG wind would be determined by the point where the ram pressure of the wind equals the pressure of the surrounding medium. A radius of $5 \mathrm{pc}$ is estimated for a canonical case (Chevalier 2005). Thus, the interaction of the SNR with the RSG wind can last for thousands of years.

Galactic core-collapse SNRs Cas A and G292.0+1.8 are likely examples that are currently interacting with the RSG wind of their progenitor (Lee et al. 2010, Lee et al. in prep.). By analyzing the X-ray emission from the outer shock regions of these SNRs with the Chandra X-ray observatory, we found that the remnants have been expanding inside a wind. For G292.0+1.8, the estimated wind density $\left(n_{\mathrm{H}}=0.1 \sim 0.3 \mathrm{~cm}^{-3}\right)$ at the current outer radius $(\sim 7.7 \mathrm{pc})$ of the remnant is consistent with a slow wind from a RSG star. For Cas A, we estimate a wind density $n_{\mathrm{H}} \sim 0.9( \pm 0.3) \mathrm{cm}^{-3}$ at the current outer radius of the remnant $(\sim 3 \mathrm{pc})$, which we interpret as a dense slow wind from a red supergiant (RSG) star. Our results suggest that the progenitor star of Cas A had an initial mass around $\sim 16 \mathrm{M}_{\odot}$, and its mass before the explosion was about $5 \mathrm{M}_{\odot}$. 


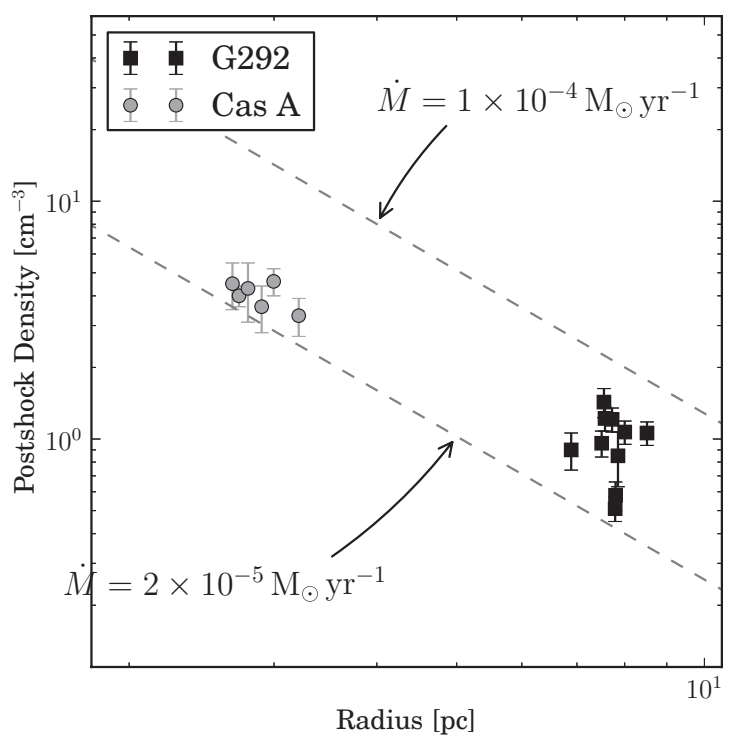

Figure 1. Densities estimated from the postshock X-ray spectra of outermost regions in Cas A and G292.0+1.8. The lines are expected postshock density for SNRs expanding inside the wind of given mass loss rate (wind velocity of $\sim 10 \mathrm{~km} \mathrm{~s}^{-1}$ is assumed).

Furthermore, the results suggest that, among the mass lost from the progenitor star $\left(\sim 11 \mathrm{M}_{\odot}\right)$, a significant amount (more than $\left.6 \mathrm{M}_{\odot}\right)$ could have been via its RSG wind.

\section{Other Young Core-Collpases SNRs}

For Young SNRs interacting with their dense RSG winds, we may expect strong Xray emission from the reverse-shocked metal rich ejecta. This seems true for Cas A and G292.0+1.8, but in other young SNRs, even though they are bright in X-ray, the ejecta signature seems weak or insignificant. A few examples are G11.2-0.3, Kes 73 and RCW 103. All 3 SNRs show NIR [Fe II] $1.64 \mu \mathrm{m}$ lines likely from their interaction with dense CSM (Koo B.-C., this Volume). An intriguing possibility is that the SNRs are interacting with dense structure around the progenitor star that were created by the star itself before the SN explosion. The nature of these SNRs needs further investigation.

\section{References}

Chevalier, R. A. 2005, ApJ, 619, 839

Chevalier, R. A. \& Emmering, R. T. 1989, ApJ, 342, L75

Lee, J., Park, S., Hughes, J. P., Slane, P. O., Gaensler, B. M., Ghavamian, P., \& Burrows, D. N. 2010, ApJ, 711, 861

Smartt, S. J., Eldridge, J. J., Crockett, R. M., \& Maund, J. R. 2009, Mon. Not. R. Astron. Soc., 395,1409

Smith, N., Li, W., Filippenko, A. V., \& Chornock, R. 2011, Mon. Not. R. Astron. Soc., 412, 1522

Sugerman, B. E. K., Crotts, A. P. S., Kunkel, W. E., Heathcote, S. R., \& Lawrence, S. S. 2005, ApJ, 627, 888 